\title{
Documentos
}

\section{La situación de Colombia y la forma como los medios de comunicación la asumen}

\author{
Mauricio Vargas
}

Quiero agradecer al Instituto de Estudios Internacionales y al Convenio Andrés Bello esta invitación. Es una excelente oportunidad de tomar unos miles de kilómetros de distancia desde Colombia para esta reflexión y es también un testimonio del interés que creo está despertando, por fortuna, en América Latina, la situación colombiana.

El tema que me corresponde es el tema de los medios de comunicación. Es sin duda uno de los temas que más polémica despierta con relación a la violencia. Todo el mundo tiene una opinión sobre la guerra, pero sobre todo, todo el mundo tiende a opinar en la forma como los medios de comunicación cubren, día a día, una guerra cada vez más difícil de cubrir, cada vez más confusa y llena de trampas para los medios de comunicación.

Desde hace un tiempo que el primer periódico de Colombia tiene, al lado de las tiras cómicas, un par de secciones muy pequeñas, más o menos históricas, pero bastante leídas, que se llaman «Hace 50 años» y «Hace 25 años». Las personas de 50 años, obvio, refieren las principales noticias de la edición del periódico del mismo día, con lo que aparece hace 50 años atrás, y las personas de 25 años hacen lo mismo con lo que sucede en este momento, y en 1974. Hay una cosa muy interesante, yo reviso las dos secciones todos los días, y mirando la de hace 25 años, existe una curiosidad. La leo todas las mañanas y parece que Colombia fuera así: pasan unas cosas interesantísimas en el Gabinete, en el deporte, en la vida económica y política, pero no hay 
noticias de guerra, no hay noticias de violencia política, éstas son muy excepcionales. Y después de varios días de revisar y ver que siempre pasaba lo mismo, que en la sección «Hace 25 años», es decir, en 1974, no aparecen noticias de violencia, me dediqué a buscar estadísticas. Y las estadísticas indican que en 1974 hubo entre 4500 y 5000 homicidios en Colombia. Hoy en día en Colombia hablamos de entre 26000 y 28000 muertes violentas.

Hay aquí una discusión entre los violentólogos, que es una profesión que Colombia ha aportado al mundo de las profesiones. Ya cuando íbamos en 4500 estábamos en un problema grande, había una guerra, pero los periódicos de Bogotá no lo registraban. Cuatro mil quinientos a 5000 homicidios al año son suficientes para que el tema sea registrado a diario y en la primera página. En Irlanda del Norte, en 25 años de guerra ha habido 3500 a 4000 muertos. Esto da unos 140 a 150 muertos al año. En Colombia, en 1974 ya hablábamos de 4500. La historia y las estadísticas nos muestran 4500 y 5000 homicidios, y el país no se consideraba en guerra. Pero tampoco se consideró en guerra en 1978 cuando ya eran 8000 ni en 1982 cuando pasamos de 12 mil. Se siguió pensando, durante mucho tiempo, que había violencia, que era un país violento pero que no había una guerra.

La explicación de que porqué los medios de comunicación, que se llamaban así pero que no lo eran, no registraban esta situación, es la siguiente. Hay varios elementos de análisis que nos permiten entender que Colombia era un país de regiones, fuertemente regionalizado desde el punto de vista de su idiosincrasia, de sus temperamentos y de su cultura. Los periódicos bogotanos, que se creían registros nacionales y no lo eran, eran de alguna manera voceros muy destacados de los líderes del Frente Nacional, voceros del Partido Liberal y del Partido Conservador y de sus dirigentes más connotados y comprometidos con el Frente Nacional.

En 1964, el Frente Nacional estaba terminando sus 16 años de alternación en la presidencia, y de pacificación. Por lo menos esa era la meta, acabar con lo que había sido la violencia liberal y conservadora. Al Frente Nacional le costaba mucho trabajo reconocer que más que pacificar a Colombia, lo que había hecho era cambiar una guerra, la liberal conservadora, por una nueva, por la guerra que da como resultado un sistema político excluyente que decidió monopolizar para dos partidos el poder político nacional, regional y local, y excluir a las demás formas, a los demás movimientos que no tuvieron bastante participación. 
Particularmente eso pasó con la izquierda. Colombia no tuvo un desarro110 de una izquierda democrática fuerte, como se dio en otros países del continente, notablemente en Chile que llegó al poder por la vía electoral. No la tuvo, entre otras cosas porque el Frente Nacional excluyó al movimiento y la única alternativa fue el móvil, la guerrilla. Nos referimos a Camilo Torres. Camilo Torres era un líder que en un sistema electoral abierto era muy posible que hubiera comprometido la situación presidencial de liberales y conservadores si hubiera tenido la oportunidad. Simplemente no podía, le estaba vedada la oportunidad de ser candidato presidencial, salvo que se inscribiera como liberal o conservador. Esa exclusión, que se expresó en los medios de comunicación y se siguió expresando al final del Frente Nacional, y que se confirmó expresando en los años siguientes, tiene muchísimo que ver con el origen de la violencia en Colombia. Mucho más que las diferencias sociales, el gran elemento que hace la diferencia en Colombia frente a otros países con iguales o peores problemas sociales y de desigualdad, es ese proceso de exclusión de una democracia formal y restringida, que margina a toda una comiente de pensamiento en ebullición en los años sesenta.

De modo que, retrospectivamente, la crítica que le podemos hacer a los medios de comunicación en Colombia, a los medios de comunicación que predominaban en el escenario de los medios de la información en 1964, hace un cuarto de siglo, es que no estaban cubriendo la guerra. Esto es exactamente la crítica contraria a la que hoy en Colombia, millones de colombianos, analistas e incluso observadores del exterior le hacen a los medios de comunicación. Hoy en día, los medios de comunicación colombianos inevitablemente se convirtieron en una arma más.

¿En qué momento empieza este cambio, que lleva a que en 25 años pasemos de una situación de no cubrir la guerra, a la situación actual, de sobrecubrimiento de la guerra, en la cual los medios hacen de la guerra un show; a una situación en la que se está multiplicando la violencia, elevando las cifras, y perturbando a las víctimas? Este cambio se produce cuando la guerra se empieza a meter en las ciudades. A mi entender, el punto que marca el quiebre, aunque ya se venía dando hace un tiempo, es la toma de la Embajada Dominicana en 1980 por el M19.

En general, la aparición del M19, una guerrilla urbana fuerte y además efectiva, necesitaba a los medios de comunicación para cumplir sus objetivos político - militares. El M19 empieza asaltando, buscando en los barrios po- 
bres. Requiere que alguien tome fotografías, que alguien los entreviste, salir en los periódicos. Como ya se ha señalado, el momento más importante es la toma de la Embajada Dominicana, que fue cubierta por los medios colombianos a diario, donde se rompió un poco la censura, o más bien la autocensura que existía en los medios. Es necesario aclarar que esta información de guerra no aparecía porque el gobierno no permitiera. Era una decisión de los propietarios de los medios, de los editores de los periódicos y de los canales de televisión, que por cierto eran públicos, y de las cadenas de radio. La representación que tenían los dueños y los editores de esos medios como voceros del Frente Nacional, los llevaba a decir que no había una guerra, y que el Frente Nacional pacificó a Colombia. Como la guerra no llegaba, aparentemente, a las ciudades, los medios de comunicación se daban ese lujo. Esta situación empieza a cambiar, justamente con la irrupción del M19 como una guerrilla, cuando se privilegia el tema de la imagen. La guerrilla se preocupaba casi tanto por la imagen como se preocupaba por el armamento y por su estrategia militar. Necesariamente los medios empiezan a ocuparse de ese tema, lo que culmina con la toma de la Embajada Dominicana.

¿Qué tipo de cosas suceden en los medios de comunicación colombianos? Primero, los medios llegan, a veces, a los lugares donde va a ocurrir la batalla instantes antes que la batalla ocurra. En 1985, el M19 se toma el tren de La Sabana, un día Domingo, día en el cual este tren está lleno de gente, de niños, de personas de la tercera edad, que van en un paseo. El M19 le avisa a un noticiero de televisión, vinculado a un sector de la Iglesia, que va a realizar esa toma. Los periodistas no le avisan a la gente y van al sitio, se suben en el tren a la hora indicada, en el lugar indicado y son testigos profesionales, y filman, graban, y entrevistan a los que llevan a cabo esa toma, una toma en que el celador del tren es asesinado por los guerrilleros.

Este suceso, a mi entender, marca una ruptura en la forma como los medios empiezan a cubrir el tema de la violencia, originándose así el debate. Claro, ¿cómo fue posible? Muere una persona. ¿Hubiera muerto si el medio de comunicación advierte? ¿Podría el medio de comunicación advertir? ¿Podrían los periodistas advertir a las autoridades? ¿Traicionaban la confianza de la fuente guerrillera? En fin, surgen todos los conflictos éticos, y periodísticos que derivan de esta situación. Pero, a partir de ahí, esta situación se volvió cada viez más común y más corriente. Digamos que, en este caso, puedo decidir como periodista que no le aviso a las autoridades. Pero le tengo que 
decir a los guerrilleros que no le voy a cubrir ese acto. Y esto se empieza a repetir, se repite muchas veces, periodistas que son informados de hechos de violencia que van a ocurrir, no que han ocurrido, y obtienen esta información privilegiada y sobrecubren. Cuando digo sobrecubrir me refiero a un cubrimiento que va más allá de lo que corresponde a la respuesta periodística lógica ante un hecho determinado.

Recuerdo otros episodios, como la toma de Florencia, realizada por el M19, que fue prácticamente transmitida en directo por narradores que parecían estar frente a un partido de fútbol, o en una carrera de ciclistas. Y muchos otros hechos, que fueron acumulando, o que fueron gestando un memorial de agravios contra la prensa en la escena de la guerrilla.

Esto no quiere decir que la prensa lo haya hecho mal. Sin duda la prensa ha hecho mucho más que esto, ha sido también una víctima. Las equivocaciones de algunos periodistas, de muchos editores, de muchos periódicos, no indican una generalización del comportamiento. En estos mismos años ha habido también en la prensa colombiana un esfuerzo de análisis que no existía, un esfuerzo por aproximarse a las causas del poder, un esfuerzo por generar un debate. Y casos particulares, en los que, además, la prensa adoptó posiciones valerosas, posiciones que significaron golpes importantes a los grupos violentos.

En diciembre de 1976 fue asesinado Guillermo Cano, de El Espectador, por el Cartel de Medellín. Acababa de publicar días antes una editorial defendiendo la extradición de narcotraficantes colombianos por Estados Unidos, la que había sido derogada por una decisión de la sala constitucional de la Corte Suprema de Justicia. Después de una ofensiva terrorista contra esa extradición, que era en ese momento la única herramienta jurídica a la que ellos le temían, el asesinato de Guillermo Cano constituyó una notificación a todos los periodistas. Nos dijeron a todos que el que opine o el que cuente algo con respecto al narcotráfico, moría. Fue además un período de muchas amenazas y la respuesta fueron la conformación de un frente de todos los medios, todos los periodistas, todas las cadenas de radio nacionales, todos los noticieros de televisión, todos los programas de información y todas las revistas. Nos pusimos de acuerdo para publicar todos, el mismo día, informes de temas que tenían que ver con el narcotráfico y los carteles.

Lo primero que hicimos fue publicar toda la información que se tenía con relación a los narcotraficantes: que hacían, qué importancia tenían, qué deli- 
tos habían cometido, cuál era la acusación que había, a veces en Colombia, a veces en Estados Unidos, y en ambos. Durante varios meses, los medios publicamos artículos sobre Pablo Escobar, sobre la conformación y la estructura interna del Cartel de Cali, o del Cartel de Medellín, sobre casos específicos como el asesinato del Ministro de Justicia don Rodrigo Jara Bailla y las pruebas que demostraban que Pablo Escobar había ordenado ese asesinato. Ante una situación que ningún medio se hubiera atrevido a publicar sólo, nos juntamos todos a tiempo. Fue un acontecimiento importante, que además marcó una reflexión de los medios sobre su papel, específicamente sobre su papel de denuncia, sobre su papel para develar a estos grupos.

Hablar de los más de 200 periodistas asesinados en el último cuarto de siglo, los cientos de periodistas que han tenido que salir producto de las amenazas, y los miles de periodistas que hemos sido amenazados en algún momento, y reiteradamente en distintas situaciones, es hablar de lo que nos corresponde como oficio. Probablemente a veces sobreactuamos en el torio lastimero de nuestros reclamos, considerando que en Colombia, finalmente, los periodistas no somos el único objetivo, ni no somos los únicos que morimos en la guerra, o que somos amenazados.

Me parece que la reflexión debe seguir girando en torno a los espejos, en torno a las equivocaciones. Hace algunas semanas se produjo la liberación de un primer grupo de secuestrados en la Iglesia La María. El Ejercito Libertador Nacional entró a una Iglesia en plena misa y secuestró a un número importante de feligreses. Cuando se liberó al primer grupo, esta liberación fue preparada por el ELN, avisada a los periodistas, en un show en transmisión en directo por televisión, en algún lugar apartado. Fueron trasladados los equipos satelitales más eficientes para esa transmisión. Viendo la transmisión esa noche, los primeros personajes en aparecer en el lugar de la liberación eran Horacio Serpa, a la sazón jefe del Partido Liberal, jefe de la oposición y muy posible candidato presidencial con altas opciones de ganar, y Don Luis Carlos Galán Sarmiento, también con grandes opciones de ser Presidente de Colombia en el próximo período. Eran las dos primeras personas que estaban allí, invitadas por la guerrilla al show de la liberación del primer grupo de personas.

Creo que ahí encontré el punto de unión entre el primer problema y el último problema. Es decir, entre la primera época de hace 25 años y esta época actual. $Y$ es que la mayor cantidad de errores por parte de los medios en el cubrimiento de la información de guerra, la hemos cometido por ir detrás de 
la dirigencia. No hemos sido autónomos. Si hace 25 años la dirigencia política decía: «aquí no hay guerra», los medios decían: «aquí no hay guerra». Pero, repito, no porque nos obligaran, era simplemente la actitud. Una región es noticia si el Presidente de la República la visita. El show de la liberación de los secuestrados lo es porque allí están en primera fila los jefes, los políticos más importantes del momento. Ese es el punto que yo creo que desmenuza cuál ha sido el papel de la prensa. Ha ido detrás, a veces detrás del gobierno de turno, a veces detrás de los militares, a veces detrás de los guerrilleros. La prensa nunca ha ido ella sola. Por fortuna, a comienzos de 1999 se empezó a romper esta tradición.

La prensa se ha demorado mucho en lograr el espacio propio que requiere para el cubrimiento de la guerra. No porque haya existido de parte de las autoridades una presión para su censura. En Colombia, censura de prensa por instrucción gubernamental, en términos generales, no ha existido desde hace por lo menos 40 años. Ha habido persuasión, ha habido autocensura, por parte de los periodistas, de los editores, y de los propietarios de los medios. $Y$ ha habido sobre todo esa actitud de llegar después de.

Creo que en esa medida los medios hemos contribuido a que en Colombia haya guerra. Porque si hubiéramos cumplido nuestra tarea en los años sesenta, si los editores de la época hubiesen ido a Urabá, a mostrar que allá estaba empezando una guerra terrible que acompañaba el boom del banano, o al sur, al Caquetá y al Guaviare, hace 25 años, a decir que los colonos estaban comenzando a sembrar coca, que había unas plantaciones de cocaína, que los colonos se estaban metiendo en eso, que la guerrilla se estaba financiando así. Debiéramos haber ido a Magdalena Medio a ver como fuerzas paramilitares se estaban consolidando en determinadas esferas, y no lo hicimos. No lo hicimos cuando fueron los políticos, o cuando fue el Estado. Los últimos en ir fuimos los medios. Y ese es el gran pecado, y esa es la gran crítica, y esa es la gran reflexión que es necesario hacer.

En 1999 esto comenzó a cambiar. Ese año, ustedes lo vieron, el 24 de octubre, la contabilización de las personas que ingresaron al parque Simón Bolívar, donde convergían las tres marchas de la paz, fue de 2700000 personas, en una ciudad que tiene 6 millones de habitantes. En Medellín hubo 1 millón, en Barranquilla hubo 400 mil, en Cali hubo 600 mil y en Bucaramanga hubo 200 mil. Se calcula que hubo entre 3 millones y medio y 4 millones de personas que marcharon en todo el país vestidas de blanco, diciéndoles a los gru- 
pos armados que querían paz. Además había un poco de carnaval en todo esto, había muñecos con la cara de Tiro Fijo, con la cara de Carlos Castaño, jefe paramilitar. Ese mismo día, entre las FARC y el Gobierno se estaban instalando las primeras negociaciones formales de paz, con agenda. Han habido otras negociaciones, otros avances, pero las FARC reconocen formalmente que están negociando, lo que tienen un valor. La exigencia de la gente, para que esa mesa de negociaciones no se rompiera, fue de dejar los secuestros, dejar las masacres, sacar a los niños del conflicto, en fin una serie de exigencias que más allá de lo que significa cada una. Los medios por primera vez se pusieron del lado de la gente, donde deberían haber estado desde un principio. La noticia con la que los medios abrieron sus emisiones esa noche fue la marcha de la paz. Eso de alguna manera me permite tener alguna esperanza, la movilización de la gente y la forma como los medios están acompañando esa noticia nos permite tener alguna esperanza de que estamos ante un proceso de paz, un proceso de negociación política que avance con las FARC, con el ELN, y con los subgrupos armados que han surgido en estos últimos años.

La economía va a decrecer este año al 3\%. Los grandes grupos económicos y sus empresas han perdido valor neto, y en buena medida esto está sucediendo por la guerra. Hay una relación entre guerra y depresión. El hecho de que los grupos económicos y la clase dirigente hayan sentido la guerra, nos despierta esperanza.

Los otros que finalmente reaccionaron fueron los Estados Unidos. Tenían una guerra ahí, a tres horas de avión y se desentendieron sistemáticamente, muy preocupados por Centroamérica, muy preocupados por Cuba. En unas conversaciones Nixon-Kissinger, en la Nación, en 1971-1972, refiriéndose a América Latina, se señalaba que en estos países era muy difícil que hubiera una democracia, eran países inmaduros para una democracia. Justificaban un poco el rebrote de gobiernos militares en el cono sur. Señalaban que el único país excepcional era Colombia. La actitud de Estados Unidos fue desconocer lo que estaba pasando en Colombia. La guerra fue desconocida no sólo para la clase dirigente colombiana, no solo para la prensa colombiana, no solo para la economía colombiana, también lo fue para los Estados Unidos. Creo que el hecho de que Estados Unidos se haya dado cuenta de este estado ha ayudado también de alguna manera.

Antes de terminar yo quería hacer algunas observaciones que no tienen mucha relación con el tema de los medios pero que son un poco lo registrado 
en la experiencia periodística frente a la guerra. $Y$ es que creo que nos hemos acostumbrado a decir una serie de cosas equivocadas en relación a la guerra colombiana. Hoy las FARC perdieron por el norte del país, por la costa atlántica, su salida al mar. Están buscando desesperadamente una salida por el Pacífico, por el lado sur de Colombia. No han podido establecer un canal permanente, como lo tenían, para recibir armamento. Han perdido mucho su poder frente al avance del militarismo. En este momento la guerrilla colombiana se abastece de armas casi exclusivamente vía Venezuela. Aparecen intercambios de armas por drogas en un proceso bastante complejo. El Ejercito de Liberación Nacional, como se ha señalado ha tenido acciones «espectaculares» este año. Acudió a esas medidas por el deterioro de su presencia militar en regiones donde había sido tradicionalmente importante, por la pérdida de su liderazgo. Perdió a su jefe tradicional y está profundamente, no dividido, pero sí tiene una tensión internacional muy complicada.

El segundo mito que es oportuno desmentir es que la guerrilla no es ninguno de los dos extremos que la derecha y la izquierda le han querido atribuir, ni son los nuevos libertadores de Colombia, como lo señala algún desprevenido servicio europeo, que habla del cura Pérez como el Cristo de Los Andes, ni son unos narcotraficantes dedicados. Son un grupo armado, fuerte, con un liderazgo y unos principios políticos que existen y prevalecen, pero profundamente corrompidos y utilizados por 40 años de guerra y por el tema del narcotráfico. Esto hay que entenderlo para entender lo que pasó en Colombia. No son ni unos guerrilleros que están construyendo una nueva libertad, ni son simplemente unos delincuentes y unos narcotraficantes con los que no hay nada que hacer. La intervención norteamericana ni es inminente ni está detrás. Algunos medios han dicho que es inminente. Algunos gobiernos han dicho que hay planes de intervención. Claro que hay planes. El SrMontecinos, en Lima se reunió con corresponsales de la prensa extranjera, en una conversación off the record, que apareció en varios periódicos, entre ellos el Clarín de Buenos Aires. Se difunde una descripción detallada de los planes que tenía el Departamento de Defensa de Estados Unidos para intervenir militarmente, incluso con tropas y con armamento en Colombia, con la ayuda de otros ejércitos, entre otros los de Ecuador y de Perú. Sin embargo, esto no quiere decir que la intervención sea inminente. Una de las tareas que tiene el Departamento de Defensa de Estados Unidos es diseñar escenarios de intervención en Colombia y en otras partes del mundo, particularmente en los lugares de 
conflicto, y Colombia es por excelencia el conflicto del hemisferio. Es el único que sobrevive después de décadas.

También hay una sobrepreocupación por el tema de la intervención de Venezuela. El gobierno de Venezuela está interviniendo de forma activa y dañina en el proceso de paz. Existe una relación de intercambio de información, de diálogo y de contactos permanente entre el gobierno de Caracas y la guerrilla colombiana, lo que es visto de manera preocupante en Colombia. Hay una insistencia del gobierno de Venezuela por la liberación de cuatro venezolanos capturados en Colombia. Fueron capturados cuando estaban reunidos con gente de las FARC en un episodio en que hay drogas y armas. Es muy importante que América Latina. siga este asunto con particular inquietud. Lo digo por fortuna, porque hablamos mucho del tema de la intervención de Estados Unidos y hablamos poco de un ejercicio de intervención muy riesgoso que se está dando desde Caracas. Además, creo que el Gobierno de Chávez y el Canciller Burelli no tienen un plan sobre Colombia, están improvisando y ante esa improvisación Colombia puede salir muy perjudicado. Las relaciones entre Colombia y Venezuela pueden salir perjudicadas.

El cuarto mito que creo oportuno desmontar es la violencia como cultura, la cultura de la violencia. Se sostiene que solamente un 5\%, 10\% o $15 \%$ de los homicidios en Colombia tienen que ver con el conflicto armado de origen político. Eso no es cierto. La revisión de las cifras nos ha obligado a revisar este concepto. En Colombia hay subdenuncias de los homicidios y hay subinvestigaciones y hay un nivel de impunidad importante. Muchos homicidios no son registrados por las autoridades como relacionados con los conflictos armados y muchos delitos de todo tipo no son registrados como delitos que se cometen en el marco del conflicto armado. En la oficina Observatorio de la Paz, se muestra con mapas una enorme coincidencia entre los homicidios llamados de delincuencia común y las zonas donde el conflicto armado está más acentuado. Lo que sucede es que, en una zona donde hay guerra, donde hay una presencia de un grupo irregular que produce una masacre para acceder a un territorio, es lógico que el resto del delito crezca a la sombra. Donde el conflicto armado se acentúa, son mucho más los valores que se pierden, son muchas más las reglas que desaparecen, de las pocas que hay. Es mucho menor la capacidad que tiene el estado de mediar otro tipo de conflictos colaterales. De modo que si se acaba con el conflicto armado sí vamos a acabar con muchos más conflictos. No es que los colombianos seamos unos 
asesinos patológicos. Es obvio que si se llega a una paz negociada con las FARC en 1,2 o 3 años va a haber mucha mano de obra violenta que va a quedar suelta, y posiblemente el secuestro aumente como acaba de pasar en Guatemala. En Guatemala no ha hecho más que crecer desde que se firmó el acuerdo de paz. Pero no por eso debemos dejar de pensar que el proceso de paz es importante, y que una negociación política con la guerrilla es importante.

Otro mito que existe, y que se ha extendido internacionalmente, es que la zona de desmilitarización favorece militarmente a la guerrilla. Creo que eso ha hecho un daño enorme. Se ha convertido en un sitio donde se ha concentrado buena parte de la tropa guerrillera. Cuando hay ataques guerrilleros, en algunas de las poblaciones al sur del país por fuera de la zona de distensión, porque obviamente la guerrilla no ataca la zona de distensión, si se retira al día siguiente, ya se sabe para dónde va la guerrilla, va para la zona de distensión. Lo que se hace es trazar la línea recta entre la población atacada y la zona de distensión, y se corta la retirada sin siquiera con la presencia de tropas. Eso produjo 45 guerrilleros muertos. Ala guerrilla no todo le ha convenido por la zona de distensión.

Con respecto al tema de la impunidad, es cierto, Colombia es un país con altísimos índices de impunidad, pero por fortuna viene en descenso. No podemos seguir hablando de una impunidad de un $95 \%$, de $90 \%$, de $85 \%$, entre otras cosas porque habíamos resuelto medir la impunidad por los casos que no conducían a una condena. Todo proceso penal que no terminara en una condena asumíamos que constituía impunidad. Aunque eso es mucha impunidad, claro, cuando un culpable no es condenado, es impunidad. La impunidad hay que medirla sobre la base de cuántos casos llegan a tener un fallo. Con respecto la justicia penal creada después de la reforma de la Constitución de 1991, en los procesos penales posteriores a 1991, entre el 50 y $55 \%$ de los casos están llegando a un fallo antes de 20 meses. Eso en Colombia es importante, se ha avanzando.

Otro mito que hoy se ha repetido muchísimo es el tema de la Unión Patriótica. Yo mismo muchísimas veces, en mis escritos, en mis columnas, lamenté, condené los asesinatos de los dirigentes de la Unión Patriótica. Pero como nos lo dijo en la Revista Semana Bernardo Jaramillo, que era el jefe de la Unión Patriótica y candidato presidencial, semanas antes de ser asesinado, él estaba consciente que a sus militantes los estaba matando la falta de claridad de sí eran guerrilleros o políticos. Esto no sucedió porque una guerrilla se 
desmovilizó, firmó la paz, formó un partido político y los mataron. Eso también ha pasado en Colombia. Pero eso no fue el caso con la Unión Patriótica. La Unión Patriótica era un partido político que estaba vinculado con una guerrilla en armas. Que más puede suceder con un militante de la Unión Patriótica si él es el representante político y lo dice y lo acepta, de un grupo armado que embosca, que mata, que secuestra. Necesariamente se vuelve también en un objetivo militar y eso fue lo que pasó. La Unión Patriótica nunca tuvo la suficiente claridad, y no la pudo tener porque las FARC no se lo permitieron. Para ser un partido político se tuvo que haber desvinculado de las FARC. De hecho, en los postulados de la Unión Patriótica estaba acordada y aceptada la combinación de las fuerzas de lucha armada y política para alcanzar los propósitos. Innecesariamente eso los convirtió en un objetivo militar. Hubo una masacre, pero no fue simplemente por intolerancia del país frente a los esfuerzos de unos hombres motivados por volverse políticos. Había una ambigüedad nunca debidamente aclarado por sus dirigentes y Bernardo Jaramillo murió consciente de eso. 